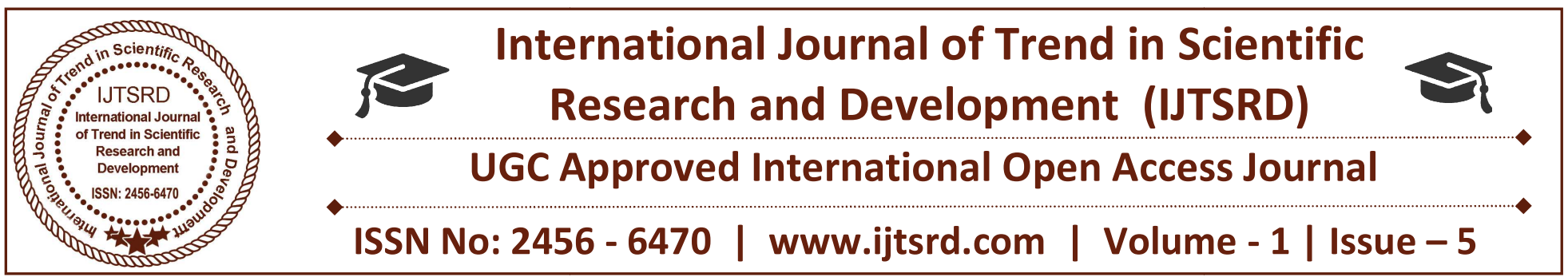

\title{
Visual and Thermal Inspection of Power Substation by Robot
}

Tushar Patil

Department of Electrical \& Electronics Engineering, AL- Falah University, Faridabad, Haryana, India

\author{
Ameen Uddin Ahmad \\ Assistant Professor, Department of Electrical \& \\ Electronic Engineering, AL-Falah University, \\ Faridabad, Haryana, India
}

\section{ABSTRACT}

Electricity sector in India is growing at rapid pace. During the current year 2017-18 (Up to 31.05.2017), the Peak Demand is about 159.6 GW and the Installed Capacity is $330.3 \mathrm{GW}$ with generation mix of Thermal (67.1\%), Hydro (13.5\%), Renewable $(17.3 \%)$ and Nuclear (2.1\%). To maintain such a big system it is necessary to develop a scheme which overcomes issue of failure of equipment in any circumstances. To overcome this failure, maintenance in power substation at regular interval has been carried out, for this auditing of power equipments temperature, testing result \& mechanism is an effective way to prevent accidents at electrical power substations. Normally inspection task is carried out by a qualified technician who normally carried out visual inspection, listen abnormal sound and use a portable thermal vision camera to measure the IR emission intensity of the equipment, thus determine its heat rate to prevent accidents, damage of equipment etc, this temperature audit \& other methods should be conducted on a regular basis to get better availability of the system. This paper presents important achievements in the field of electric power substation equipment's inspection by robot. Mobile Robot has a flexible thermal vision \& normal camera and is designed for automated auditing of substation power equipment temperature and any abnormality.

Keywords: Robot behavior, equipment inspection robot, positioning identification, power substation

\section{INTRODUCTION}

Long with the quick development of the electric power sector, there is a demand of power due to high dependency of people on electrical equipment. In order to minimize failures of power and avoid the huge economic losses caused by power outages, transmission line and substation equipment ought to be regularly inspected for detecting defects as soon as possible to arrange the maintenance plan. Periodic inspection and maintenance (PiM) shall be performed to verify proper system operation and general maintenance day by day or as per breakdown. Periodic inspection and maintenance (PiM) helps ensure the quality of an end user's electricity by enhancing the reliability of substations. Periodic inspection and maintenance (PiM) accomplishes this increased reliability by monitoring equipment over time in order to isolate conditions that indicate impending failure. The goal is to determine whether corrective action is required and, if so, to take that action before equipment fails. It is well known that, as an essential part of modern power systems and future smart grid, the safe and stable operation \& maintenance of the substation equipment plays a very important role in the entire power system.

Substation equipment inspection is an important means for operation and maintenance, which can ensure the secure working of the electric grid, improve power grid to run economically and afford good service for user .There are roughly speaking, two common methods of power equipment inspection, which are foot patrol and inspection by robot. 


\subsection{Problem Definition}

Now days, substation equipments are inspected by workers in our country. It not only costs much manpower and material resources, but is also inefficient and insecure. In order to resolve this contradiction and take in to account the traditional operating model of substation, mobile robot system for monitoring equipment in power substations is a much better choice to solve the above problems. In the foot patrol inspection, usually a team of two people traverse the substation along the scheduled route and inspect the power equipment such as power transformer, circuit breaker, current transformer, capacitor voltage/potential transformer, checked power transformer temperature, circuit breaker air pressure \& SF6 gas pressure and so on parameter required for safe operation. This kind of inspection is slow, tedious, monotonous and subjective so that even larger faults can sometimes be neglected. In order to improve the reliability, safety and intelligence of power substation and accelerate the realization of unattended substation, the problem of automated inspection of power substation equipment has become an important issue of the electric power system. With the development of robot technology and visual inspection methods which are carried out with the help of binoculars and sometimes with Infrared Ray (IR) and corona detection cameras, an available solution is to develop autonomous mobile robots, and then develop an automatic inspection approach that would be more accurate, less expensive and at least as fast as foot patrol inspection. Besides, another kind of power facilities inspection mobile robot is also developed to inspect and maintain power transmission line, and the technology of power substation equipment inspection robot can also be applied to the other hazardous environment. In comparison to the substation equipment foot patrol inspection, inspection by mobile robots would be less objective, with better inspection accuracy, better price performance, greater market space and development prospects. In this paper, we only focus on discussing power substation inspection robot.

\subsection{Objective of Thesis}

Power substation inspection robot plays an important role in the field of electricity power facilities application special robot research. There is considerable research on the technology of power substation inspection robot. In the research work on power substation inspection robot is composed of mobile vehicle platform, testing sensors such as IR and corona detection cameras, the control and communication unit, and so on, have been discussed. The inspection system based on the robot have sensor of visible Charge-coupled Device (CCD) also required, which help people to achieve better reliability. In India these technology was not on peak, but a regular study has been carried out by many research centre. Following fig 1.1 shows example of robot which have camera over it and controlled by a operator manually.

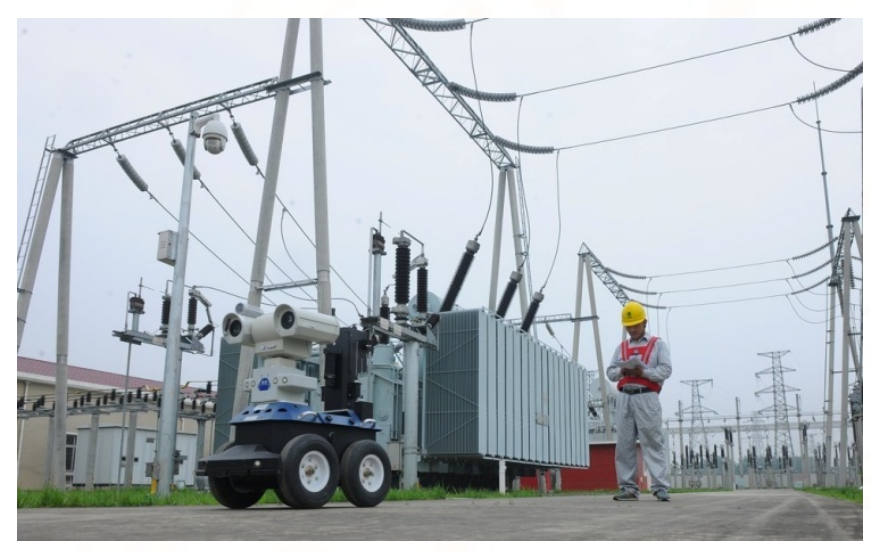

Fig-1.1: Power substation robot operated manually by operator

\section{TYPES OF INSPECTION}

This section briefly describes the inspection tasks, which need to be undertaken for power substation equipment, and it involves the most common robotic inspection technology.

\subsection{Substation Equipment Inspection Tasks:}

Power substation equipment inspection is an important technology to ensure the safe operation of power equipments. According to the research, the substation equipment inspection can be mainly classified into four types, which are as follows:

\subsubsection{Normal inspection (including handover inspection) \\ 2.1.2 Inspection after light out at night \\ 2.1.3 Comprehensive inspection (Standardized 2inspection) \\ 2.1.4 Special Inspection}




\subsection{Power Substation Inspection By Mobile Robot}

Technology of power substation inspection robot is one of the frontier research topics in the field of robot research, which is a combination of many disciplines, such as mechanics, high voltage insulation technology, electronics, control theory, computer science and artificial intelligence and so on. And the robots, assisting or replacing staffs, detect thermal defects, reading different parameter and abnormalities and other issues of substation equipment. Then the fault accidents and potential risk data are provided to staffs. However, on account of the complex working environment of the power substation inspection robot, there are some key technologies that are difficult to achieve breakthroughs. These key technologies are as follows.

\subsubsection{Design requirements of inspection robot}

2.2.2 System Architecture of Power Substation Inspection Robot

2.2.3 Construction of Power Substation Inspection Robot

2.2.4 Control of Power Substation Inspection Robot

\section{BEHAVIOUR OF INSPECTION ROBOT}

The substation inspection robot, which works in the environment of the substation with strong electromagnetic interference, is different from the general industrial robot. And it is difficult to achieve the control of autonomous motion, avoiding obstacle and navigation. In order to improve the intelligent level of inspection robot, there are some technical problems to be solved, which are as follows:

\subsection{Behavior and navigation of power substation inspection robot}

Navigation Technology of Power Substation Inspection Robot - Inspection robots move and inspect on the road of substation equipment inspection area which is a kind of unstructured environment of strong electromagnetic fields and equipment shades. Furthermore, the substation environment scope is large and the ground natural features that the computer vision can recognize are little (see Fig.1.2).

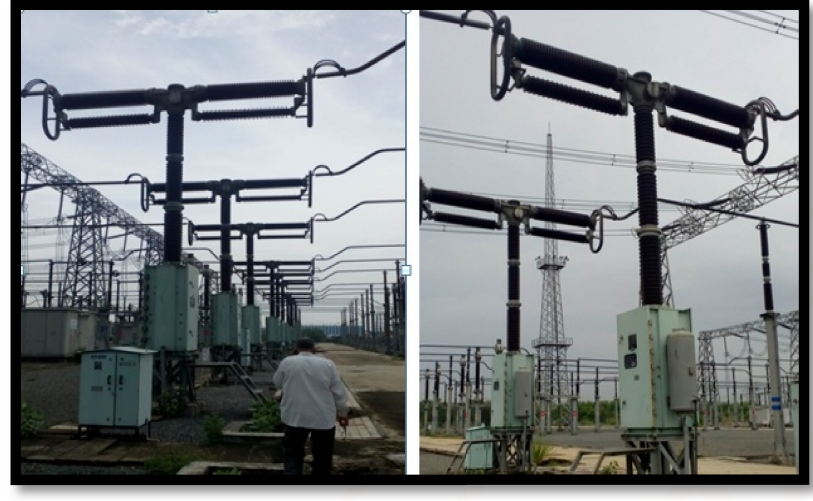

(A) Large Range

(B) Short Range

Fig -1.2: Power substation environment

Currently, there are so many methods employed in inspection robot navigation and positioning in substation, such as magnetic track guidance and Radio Frequency Identification Devices (RFID) positioning, GPS navigation, laser positioning navigation and visual navigation. Same has been discussed below:

$>$ Magnetic Track Guidance and RFID Positioning

$>$ GPS Navigation

$>$ Positioning Navigation

$>$ Visual Navigation

\subsection{Equipment Detection Technology Based On Power Substation Inspection Robot}

Power substation equipment detection system is an important part of the inspection robot system. With the development of the substation automation degree, substation Remote Maintenance System (RMS) has been realized in some areas, equipment inspection is still an indispensable means of substation operation and maintenance. And the detection system of the inspection robot should be able to automatically inspect the appearance image of equipment, running equipment sound, the temperature of the equipment joint and other information of the substation running equipment, and identify the status of the preparation.

Detection of Thermal Defect in Equipment by Infrared - The infrared thermal imager can turn infrared radiation of substation equipment into heat distribution image using infrared technology, which not only can measure the temperature without contact, but also can display two-dimensional distribution and changes of equipment surface temperature in real-time as shown in fig. 1.3. 


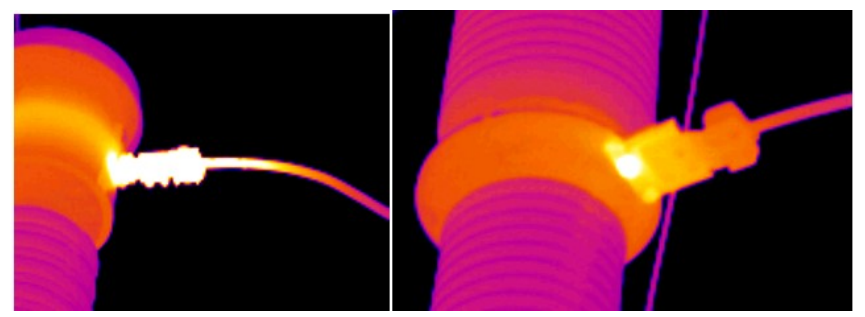

Fig -1.3: Power substation environment

\section{TECHNOLOGY OF INSPECTION ROBOT}

Because the robot is working in the environment of the substation, the robot must not only ensure the safety of the robot itself, but also cannot bring bad influence to the operation of the substation equipment. Otherwise, potential faults may bring great harm to the power grid. Here Paragraph comes content here. The robot operation mode management module can implement the real-time motion control and inspection task management by means of autonomous control or remote control, and the substation equipment visible light image, infrared image/video.

\subsection{Wireless technology for inspection robot}

In order to ensure the safe and stable operation of the robot, the Real-time Transport Protocol (RTP) bidirection control information link under wireless communication was sent from substation site to operator screen or in storage as shown in fig. 1.4.

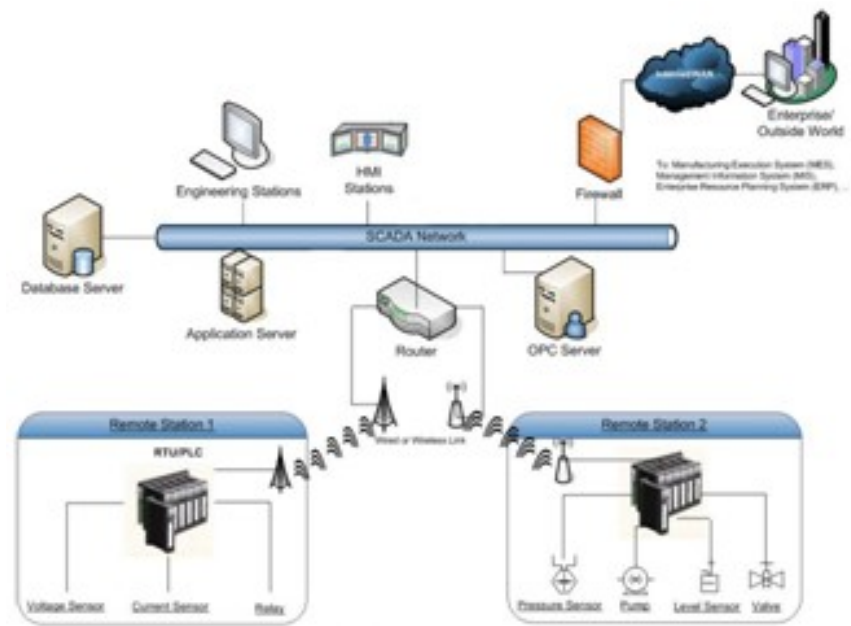

Fig-1.4: Power substation SCADA architecture

\subsection{Operation Technology for Inspection Robot}

In general, motion information of inspection robot, which includes the dynamic angle displacement, dynamic angle velocity, dynamic angle acceleration, can be detected by its onboard sensors, and are sent to the background monitoring system and provided to the operator with the robot operating base. According to the working environment and working characteristics of the robot, the inspection robot should be operated by remote control in the following situations:

$>$ Robot faults, such as power shortage, sensors, etc.

$>$ The special inspection tasks, which are not, included in the autonomous inspection tasks as shown in fig. 1.5.

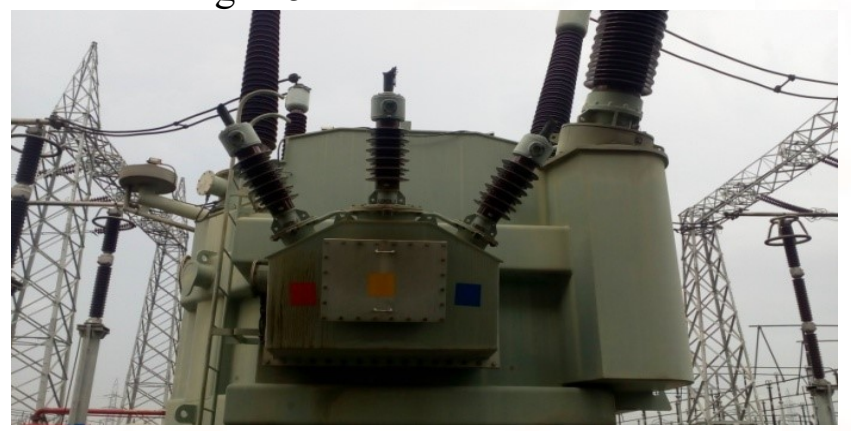

Fig-1.5: Special vision of Tertiary winding of Power Transformer by inspection robot using remote

\subsection{Background Monitoring System of Inspection Robot}

In order to ensure the safety of the inspection robot and its detection operation, the inspection robot process need to be monitored by operators in real time, then the operators and inspection robot need the information exchange interface, which can also provide the platform for the analysis and early warning of substation equipment. The background monitoring system, which includes the monitoring system, analysis and early warning system, is such an information interaction platform between inspection robot and the operators as shown in fig. 1.6. Pointer shows highest temperature on top of bushing right side i.e. $104^{\circ} \mathrm{C}$ temp detect by robot.

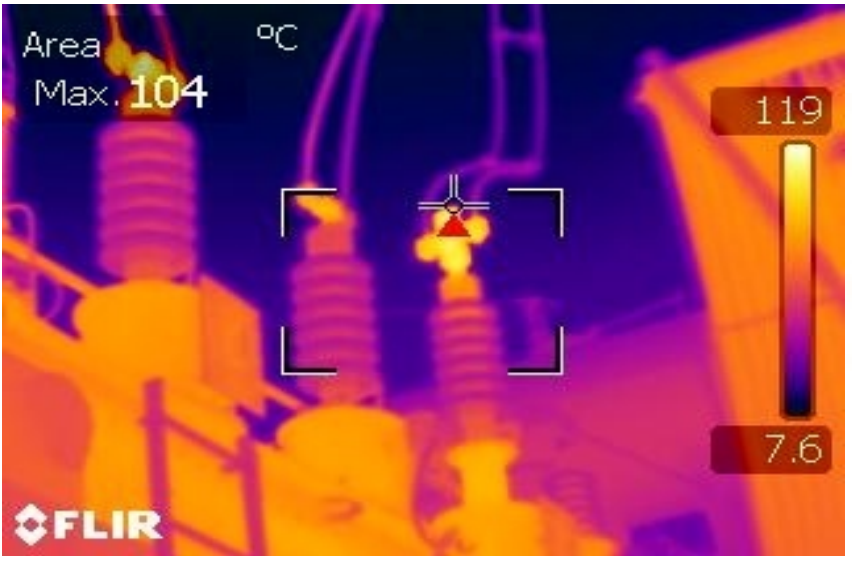

Fig-1.6: Analysis of hot spot by inspection robot 


\section{CONCLUSIONS}

With the constantly growing and in-depth research on power substation inspection robot in Indian substation, inspection robot study was done. Along with the application of inspection robot in substation, it can not only reduce the labor intensity of staffs, but also improve the work efficiency, and the corresponding frequency of inspection by robot can be set arbitrarily. However, there are still a number of key technologies to be achieved breakthroughs. In order to improve the development and application of inspection robot system, further development and research of power substation inspection robot should focus on the following issues.

Mechanism optimization

$>$ Multi-sensor information fusion technology

$>$ Remote centralized control system

$>$ Integrated substation automation technology

\section{ACKNOWLEDGEMENT}

There is always a sense of gratitude which one expresses to other for the help and timely services they render during all phases of life. I too would like to do it as I really wish to express my gratitude towards all those who have been helpful to me. I am thankful to assistant professor Ameen Uddin Ahmad (Electrical \& Electronic Engineering, dissertation Supervisor) for providing me the opportunity to prepare a project in power system.

\section{REFERENCES}

1) J. K. C. Pinto M. Masuda L. C. Magrini J. A. Jardini. Mobile robot for hot spot monitoring in electric power substation. Transmission and Distribution Conference and Exposition 2008.

2) J. K. C. Pinto M. Masuda L. C. Magrini J. A. Jardini. Mobile robot for hot spot monitoring in electric power substation. Transmission and Distribution Conference and Exposition 2008, pp. $1-5$.

3) Li Shengfang Hou Xingzhe. Research on the AGV Based Robot System Used in Substation Inspection. 2006 International Conference on Power System Technology, pp. 1-4.
4) J. K. C. Pinto M. Masuda L. C. Magrini J. A. Jardini. Mobile robot for hot spot monitoring in electric power substation. Transmission and Distribution Conference and Exposition 2008, pp. $1-5$.

5) Rui Guo Peng Xiao Lei Han Xueqi Cheng. GPS and DR Integration for Robot Navigation in Substation Environments. IEEE International Conference on Information and Automation. Harbin China 2010.

6) Ou Qing-Li, He Ke-Zhong. Research on key techniques and development of outdoor intelligent autonomous mobile robot. Robot, 2000, 22(6): $519-526$. 